\title{
La trampa entomológica Gravid-Aedes Trap, como una alternativa para el monitoreo del mosquito Aedes aegypti, vector del dengue, en las islas Galápagos
}

The Gravid-Aedes Trap as an alternative for surveillance of the Aedes aegypti" mosquito, the vector of dengue on the Gallapagos Isllands

\section{Luis Renato León Villalba - Andrés Ricardo Carrazco Montalvo - Ivis Alexi Llerena Martillo - Agustin Hinojosa Gavilanes}


Luis Renato León Villalba

Universidad San Francisco de Quito USFQ

Laboratorio de Entomología Médica \& Medicina Tropical (LEMMT),

Campus Cumbayá

Quito 170901, Ecuador

rleon@usfa.edu.ec

https://orcid.org/0000-0002-1909-8445

Andrés Ricardo Carrazco Montalvo

Universidad San Francisco de Quito USFQ

Laboratorio de Entomología Médica \& Medicina Tropical (LEMMT),

Campus Cumbayá

Quito 170901, Ecuador

andres.carrazco@hotmail.com

https://orcid.org/0000-0002-3581-7761

Ivis Alexi Llerena Martillo

Consultor independiente

Quito 200101, Ecuador ivis_1@hotmail.es

https://orcid.org/0000-0001-8255-0505

Agustin Hinojosa Gavilanes

Universidad Federal de Minas Gerais Laboratorio de Innovación Tecnológica y Emprendimiento

en Control de Vectores (Lintec)

31330530-Belo Horizonte, Minas Gerais, Brasil agustinhoo@hotmail.com

https://orcid.org/0000-0002-7608-7902 


\section{Resumen}

El mosquito Aedes aegypti es un insecto díptero de importancia médica y es vector de varios arbovirus, entre ellos el dengue, el chikungunya y el Zika. Esta especie es diurna y está adaptada a vivir cerca del ser humano en ambientes urbanos. En 1988 ocurrió el primer brote epidémico de dengue en Ecuador. Desde entonces, es endémico y ocasiona picos de transmisión cada cuatro o cinco años. En las islas Galápagos, el dengue fue introducido en 2002, cuando se reportaron los primeros casos en la isla Santa Cruz. Luego, un brote epidémico de magnitud ocurrió en 2010 en la isla San Cristóbal y, desde entonces, se reportan casos esporádicos cada año. Pocos casos de chikungunya y de Zika también han sido reportados. En el archipiélago, las medidas para controlar a la población de mosquitos se restringen al control vectorial mediante abatización que elimina las larvas, y fumigación con el insecticida Deltametrina para matar a los adultos. Actualmente, no hay un sistema de monitoreo rutinario de las poblaciones de mosquitos, que se considera crucial para evaluar los métodos de control más adecuados y adoptar las medidas más convenientes para combatir al mosquito vector y disminuir la transmisión de enfermedades. Se presenta la trampa GravidAedes Trap (GAT) como una herramienta de monitoreo pasivo y fácil de usar. Su precio es asequible y no requiere de una fuente de electricidad para funcionar. Estudios de evaluación en Brasil muestran la utilidad de esta trampa para el monitoreo y también para el control vectorial. Se reporta un estudio piloto con 10 trampas GAT que se colocaron en Puerto Baquerizo Moreno, en la isla San Cristóbal, y en Puerto Ayora, en Santa Cruz, para monitorear mosquitos. Los resultados preliminares sugieren que esta metodología puede ser efectiva para colectar Aedes aegypti y, por tanto, de potencial utilidad para complementar las estrategias de control vectorial que se llevan a cabo rutinariamente en las islas Galápagos. Otras especies de mosquitos colectadas fueron Culex quinquefasciatus y Aedes taeniorhynchus. Son necesarios estudios a mayor escala para seguir evaluando la utilidad de esta nueva trampa.

Palabras clave: monitoreo vectorial, archipiélago de Galápagos, trampas GAT, fiebre del dengue, chikungunya, Zika. 


\section{Abstract}

The Aedes aegypti mosquito is a two-winged insect of medical importance as it is the main vector of several arboviruses including dengue, chikungunya and Zika. This species is diurnal and is adapted to live close to the human population in urban settings. In 1988, the first dengue outbreak occurred in Ecuador. Since then, dengue has been endemic, causing peaks of transmission every four or five years. On the Galapagos Islands, this species was introduced in 2002 when the first cases were reported on Santa Cruz Island. Subsequently, a major epidemic outbreak occurred in 2010 on San Cristóbal Island, and since then, sporadic cases have occurred every year. A few cases of chikungunya and Zika have also been reported in past years. In the archipelago, the activities to reduce the mosquito populations have been restricted to vector control through abatization campaigns, to kill the mosquito larva, and fumigation with the insecticide Deltamethrin, to kill the adults. Mosquito surveillance initiatives have not been developed; nevertheless, they are crucial to be able to evaluate the best vector control strategies and choose the most adequate measures to eliminate the vector mosquitoes and reduce disease transmission. The Gravid-Aedes Trap is presented as an easy-to-use, passive monitoring tool for vector surveillance. It is affordable and does not require electricity. Evaluation studies in Brazil have shown the usefulness of this trap to conduct vector surveillance and vector control. A pilot study is herein reported with 10 GAT traps that were placed in Puerto Baquerizo Moreno, San Cristóbal Island and in Puerto Ayora, Santa Cruz Island to carry out mosquito surveillance. Preliminary results suggest that the GAT traps may be effective for collecting Aedes aegypti mosquitoes and thus of potential use to complement the vector control strategies that are routinely carried out on the Galapagos Islands. Other mosquito species collected were Culex quinquefasciatus and Aedes taeniorhynchus. Future large-scale studies are necessary to further evaluate the usefulness of this new trap.

Keywords: vector surveillance, Galapagos archipelago, GAT traps, dengue fever, chikungunya, Zika 


\section{Resumo}

O mosquito Aedes aegypti é um inseto díptero de importância médica por ser vetor de vários arbovírus, incluindo o vírus da dengue, chikungunya e Zika. Esta espécie é diurna e está adaptada a viver em ambientes urbanos. Em 1988, o primeiro surto de dengue ocorreu no Equador. Desde então, a dengue é endêmica, causando picos de transmissão a cada quatro ou cinco anos. Em Galápagos, esta espécie foi introduzida em 2002, os primeiros casos foram registrados na llha de Santa Cruz. Posteriormente, um surto epidêmico foi registrado em 2010 na llha de San Cristóbal e desde então, casos esporádicos foram relatados a cada ano. Alguns casos de chikungunhya e Zika também foram descritos. No arquipélago, as atividades de controle da populações de mosquitos restringem-se ao controle do vetor, por meio da eliminação de larvas e da fumigação com deltametrina para controle populacional dos adultos. Atualmente, a falta um monitoramento constante para com as populações do mosquito, fundamental para avaliar os métodos de controle mais adequados e adotar as medidas mais convenientes para combater o mosquito vetor e reduzir a transmissão de doenças.A Gravid-Aedes Trap é uma ferramenta de monitoramento passiva e de fácil de uso, seu preço é acessível e não requer fonte de energia elétrica para seu funcionamento. Estudos de avaliação no Brasil mostraram a utilidade desta armadilha para monitoramento e também para controle de vetores. Um estudo piloto foi realizado utilizando 10 armadilhas GAT, instaladas em Puerto Baquerizo Moreno(ilha de San Cristóbal) e em Puerto Ayora (ilha de Santa Cruz) para monitoramento de mosquitos. Os resultados preliminares sugerem que as armadilhas GAT podem ser eficazes para coletar Aedes aegypti e, portanto, com potencial para complementar e direcionar estratégias de controle de vetores que já são realizadas nas Ilhas Galápagos. Outras espécies coletadas foram Culex quinquefasciatus e Aedes taeniorhynchus. Estudos de avaliação em larga escala são necessários para avaliar ainda mais a utilidade dessa armadilha recémdesenvolvida.

Palavras-chave: monitoramento de vetores, arquipélago de Galápagos, armadilhas GAT, dengue, chikungunya, Zika 


\section{Introducción}

\section{Los vectores del dengue}

Los mosquitos (familia Culicidae) son pequeños insectos dípteros (que tienen dos alas) de patas largas y partes bucales adaptadas para picar (solamente en las hembras) y alimentarse de sangre (hematofagia). Son llamados vulgarmente "zancudos", y uno de los principales grupos de insectos que pueden transmitir enfermedades mediante su picadura (vectores). Muy pocas especies son responsables de transmitir la fiebre del dengue. Entre las más importantes se encuentra el Aedes aegypti, zancudo originalmente conocido como el "mosquito de la fiebre amarilla", por ser capaz de transmitir también esta peligrosa arbovirosis. Esta especie fue importada desde África a las Américas entre los siglos XVI y XVIII [1], y se distribuyó rápidamente en zonas cálidas a nivel global. Se constituye como uno de los vectores más ampliamente distribuidos y agresivos del planeta. Otra especie transmisora de la fiebre del dengue es el Ae. albopictus, también conocido como el "mosquito tigre asiático", que proviene de Asia y fue introducido en las Américas a mediados de los ochenta [2,3]. Ambas especies también han sido asociadas a la transmisión del chikungunya y del Zika, enfermedades arbovirales que en esta última década han causado importantes brotes epidémicos en las Américas [4].

En general, en los mosquitos, solamente las hembras se alimentan de sangre, que utilizan como fuente energética para el desarrollo de los huevos. Por tanto, exclusivamente las hembras son vectores; al contrario, los machos se alimentan de la savia de las plantas y no transmiten enfermedades. Los mosquitos tienen estadios inmaduros de vida acuática (larvas y pupas), por lo que su ciclo de vida se relaciona con la presencia de agua. La hembra busca recipientes con agua para colocar sus huevos. De estos, emergen larvas que crecen, se desarrollan y pasan por cuatro estadios antes de transformarse en pupas, un estadio de aproximadamente dos días de transformación, para formar el mosquito adulto. Los adultos eclosionan en el agua, estiran sus alas, salen volando y se dispersan rápidamente.

El Ae. aegypti es un mosquito urbano que vive cerca de la población humana y se reproduce en tanques con agua almacenada, en floreros y recipientes con agua desatendida o con agua de lluvia abandonados, por lo que su reproducción está asociada, sobre todo, con zonas marginales carentes de alcantarillado y agua 
potable. Ante la falta de estos servicios públicos, la población se ve obligada a adquirir agua de otras fuentes, que almacena en lavanderías y tanques bajos, para el posterior uso en quehaceres domésticos [5]. Esta especie es principalmente diurna y tiene mayor actividad a media mañana y poco antes del anochecer. En general, los mosquitos no se desplazan a más de $100 \mathrm{~m}$, aunque si la hembra no encuentra un lugar adecuado, puede volar hasta $3 \mathrm{~km}$ hasta localizar un sitio con agua para la ovoposición (colocar los huevos).

Un mosquito que se encuentra en una determinada casa proviene de la misma vivienda o de viviendas vecinas, por lo que su presencia se considera un problema de salud pública comunitario; no depende exclusivamente del cuidado que tengan los habitantes de una casa, sino también del de los vecinos, que, si mantienen agua destapada y desatendida, sea dentro del domicilio o en el patio (peridomicilio), pueden favorecer los criaderos de mosquitos y la dispersión de estos a otras viviendas de la manzana o del barrio. Para que un mosquito se infecte, debe haber picado a una persona infectada con el virus del dengue, a quien se considera el hospedero/reservorio, dentro del ciclo de vida de este arbovirus. Los mosquitos infectados, en cambio, son los vectores del virus y pueden subsecuentemente picar y transmitirlo a otra(s) persona(s).

\section{Presencia del Aedes aegypti en el Ecuador y en las islas Galápagos}

En las Américas, el Ae. aegypti posiblemente se introdujo mucho antes del siglo XX. La historia se refiere a posibles casos de dengue o chikungunya desde el siglo XVII [6]. En el Ecuador, la lucha contra el Ae. aegypti se inició a principios del siglo XX, en 1918, cuando el Dr. Michael Connors aplicó el sistema Gorgas para combatir al mosquito. Para ese entonces, las iniciativas de control vectorial se lideraban desde otros países. Hacia 1919, gracias a esta exitosa actividad de control, se reportó el último caso de fiebre amarilla de transmisión urbana en Guayaquil, en donde el Ae. aegypti era el mosquito vector de este peligroso virus [7]. En 1947, la Fundación Rockefeller retiró su participación del Plan Continental de Erradicación del Mosquito de la Fiebre Amarilla, y la Organización Panamericana

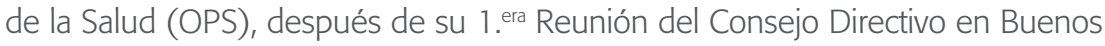
Aires, tomó el liderazgo del control vectorial en las Américas.

El Ecuador fue declarado libre del Ae. aegypti en 1958, gracias a un intenso programa que, entre otras estrategias, usó masivamente al insecticida DDT. Sin embargo, en 1975, las poblaciones de mosquitos se recuperaron y se 
establecieron nuevamente en el país, y en 1988, se produjo el primer brote significativo de dengue [8]. El virus del dengue se estableció de forma endémica desde entonces y ha producido brotes epidémicos regularmente cada cuatro 0 cinco años [9]. El último brote significativo ocurrió en 2015, con más de 42,000 casos reportados. También los brotes epidémicos de chikungunya y Zika fueron relevantes en años pasados [10].

La introducción del mosquito tigre asiático es más reciente y, a pesar de estar presente en las Américas desde los ochenta, fue reportado por primera vez en el Ecuador en Guayaquil, en la provincia del Guayas, en 2017 [11]. Hasta el momento, no hay más información publicada sobre esta especie en el Ecuador, y es posible que en la actualidad ya se encuentre disperso en otras provincias del país.

En las islas Galápagos, los primeros registros del Ae. aegypti datan de 2001 [12]. Su presencia en Puerto Ayora, en la isla Santa Cruz, se confirmó con los primeros casos de dengue en 2002. Desde esa época, la fiebre del dengue se ha vuelto endémica en esta isla, con casos esporádicos reportados cada año. Recién en 2010 se conoció la expansión a otras islas, cuando en Puerto Baquerizo Moreno, en la isla San Cristóbal, ocurrió el brote epidémico de dengue más significativo de las Galápagos hasta la actualidad, con más de 900 casos [13]. Desde entonces, el dengue se ha mantenido endémico en ambas islas, con casos esporádicos, especialmente provenientes de Santa Cruz.

Los estudios del Laboratorio de Entomología Médica \& Medicina Tropical (LEMMT), de la Universidad San Francisco de Quito (USFQ), sobre el mosquito Ae. aegypti en las Galápagos, iniciaron en 2004, cuando, en colaboración con la Universidad Estatal de Nueva York (SUNY-UMU), se llevó a cabo un estudio entomológico que confirmó la persistencia de esta especie vector del dengue en ambas islas. Los datos de un monitoreo transversal realizado indicaron altos índices de presencia del Ae. Aegypti, sobre todo en San Cristóbal, lo que alertó del riesgo de la población de presentar nuevos brotes epidémicos de dengue [8].

Nuevos estudios longitudinales en 2017 y 2018 revelaron la persistencia del vector durante todo el año en ambas islas, y sugirieron que la mayor cantidad de mosquitos se encontraba en Puerto Ayora [14]. Estos resultados corroboraron datos del programa de control vectorial del Ministerio de Salud Pública del Ecuador (MSP) y coincidieron con el criterio de que los habitantes de Santa 
Cruz tenían mayor riesgo de adquirir la enfermedad. Es interesante anotar que en este estudio también se realizaron monitoreos entomológicos en otras islas habitadas: Isabela y Floreana. Los monitoreos entomológicos alertaron de la presencia de Ae. aegypti en Isabela, lo que corroboraba el primer reporte de la presencia de esta especie en esta isla en capturas realizadas en 2014 [15]. Estos hallazgos evidenciaron el riesgo de transmisión local del dengue y cualquier otra enfermedad transmitida por Ae. aegypti en Isabela. En marzo y abril de 2020, se notificaron los primeros cuatro casos de dengue de transmisión local en Isabela, lo que alertó de la persistencia del mosquito vector en la isla y promovió el control vectorial en Puerto Villamil por parte de las autoridades del MSP (Información directa del Distrito de Salud en San Cristóbal).

\section{El control vectorial y perspectivas de uso de la trampa GAT}

Las principales actividades que se realizan en las islas Galápagos para combatir al mosquito Ae. aegypti se enfocan sobre todo en control vectorial, que rutinariamente se distribuye entre (a) un programa de abatización que consiste en colocar el larvicida Temephos, comercialmente conocido como Abate ${ }^{\circledR}$, en lavanderías y tanques con agua para eliminar los criaderos de larvas de mosquitos, y (b) un programa de fumigación de las casas, bajo requerimiento de los moradores, con el insecticida Deltametrina que se rocía a baja densidad, mediante bombas de fumigación, para eliminar a los mosquitos adultos. Aunque ambas actividades son cruciales para controlar las poblaciones de mosquitos y la transmisión de enfermedades, el monitoreo rutinario y durante todo el año de las poblaciones de mosquitos es clave para evaluar el tamaño de las poblaciones y la efectividad de las medidas de control. Esta información también permite adaptarlas de acuerdo con el resultado del monitoreo, para que sean más efectivas y minimizar o suspender la transmisión de enfermedades vectoriales como el dengue, el chikungunya y el Zika.

Los índices entomológicos de casa (número de casas positivas para Ae. aegypti por cada 100 viviendas), contenedor (número de contenedores positivos por cada 100 recipientes examinados), y de Breteau (número de contenedores positivos para Ae. aegypti por cada 100 casas examinadas) han sido históricamente referencia para monitorear las poblaciones de estadios inmaduros del Ae. aegypti y predecir el riesgo de transmisión. Posteriormente se han utilizado los índices de pupas. Sin embargo, estos índices han resultado muy poco eficientes en esta tarea, pues no todas las larvas o pupas 
sobreviven a adultos y no todas emergen a hembras de mosquito, que son las únicas transmisoras. En esta última década, los índices de adultos que cuantifican el número de mosquitos adultos por espacio físico sean por vivienda, barrio o zona geográfica determinada, han demostrado ser mejores herramientas para predecir el riesgo de transmisión [16]. Para la captura de los adultos del Ae. aegypti, se han desarrollado diferentes trampas entomológicas y métodos de colecta; entre los más importantes, se encuentran las trampas centinela BG y los aspiradores entomológicos Prokopack. Estos métodos, aunque con variaciones, se basan en el uso de una pequeña hélice que, acoplada a un motor, pueda aspirar los mosquitos a un recipiente colector. Aunque los métodos son efectivos, requieren de una fuente de energía eléctrica proveniente de corriente eléctrica de las casas o de una batería, lo que restringe su uso a zonas con luz eléctrica o el acceso a esta, para recargar las baterías. Adicionalmente, se necesita personal técnico entrenado y largas jornadas de trabajo para realizar las colectas.

Ante esta problemática, se han desarrollado otras trampas que no requieran de una fuente de poder y puedan colectar pasivamente los mosquitos en el tiempo sin la necesidad de personal. Este es el caso de la trampa Gravid Aedes trap (GAT), desarrollada por el laboratorio Lintec, en la Universidad Federal de Minas Gerais (UFMG), en el Brasil [17]. La trampa GAT está bajo continua evaluación; sin embargo, estudios realizados corroboran su efectividad para el mejor monitoreo e incluso controlar las poblaciones de mosquitos [18, 19].

\section{La trampa GAT y su funcionamiento}

La trampa GAT pretende simular el ambiente y las condiciones naturales en las que se desarrollaría un criadero de mosquitos en una zona urbana (Figura 1a). La trampa consiste en un depósito de plástico negro que se complementa con un segundo, a manera de tapa, que, al ensamblarse, arma un recipiente cerrado, excepto por una boca en la parte superior, por donde ingresarían los mosquitos. Al fondo del recipiente se coloca agua, por arriba de la cual se ajusta un anillo plástico; este sostiene una malla circular que calza horizontalmente sobre la superficie del agua (Figura 1b). Sobre la malla, se coloca un atrayente químico. Del techo de la trampa, por la parte interna, se cuelga de un gancho plástico un trozo de tela impregnada con insecticida (Deltametrina), que mata a los mosquitos. 

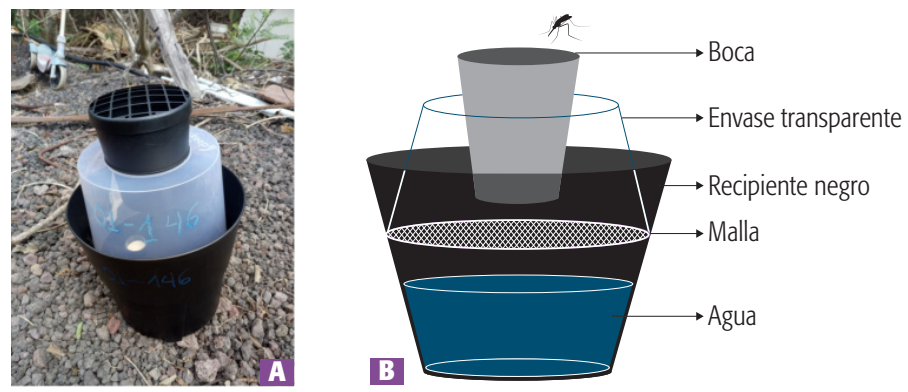

FIGURA 1. Trampa GAT y su funcionamiento. (a) Fotografía de la trampa Gravid AedesTrap (GAT). (b) Componentes de la trampa: boca por donde entran los mosquitos, envase transparente a manera de tapa, recipiente negro, malla, agua. Tomado de Eiras [17].

La trampa está diseñada para atraer a los mosquitos hembra que, después de una alimentación de sangre de sus víctimas, buscan un lugar oscuro y con agua para depositar sus huevos. Para reproducirse, las hembras son perseguidas por los mosquitos macho, por lo que la trampa puede ser efectiva para colectar ambos sexos. La trampa es fácil de usar, limpiar y manipular, y no necesita de electricidad, por lo que se la puede colocar en lugares remotos sin energía eléctrica, y es de bajo costo. Puede permanecer por semanas atrapando mosquitos sin necesitar de mayor mantenimiento; sin embargo, con fines de investigación y monitoreo entomológico, se recomienda recoger las muestras de mosquitos semanal o quincenalmente.

En esta publicación, se resume y describe información recopilada respecto al mosquito Ae. aegypti, su presencia y control en las islas Galápagos. Se expone y presenta a la trampa GAT como una alternativa para implementar el monitoreo vectorial en las islas, y se publican los resultados preliminares de un estudio piloto de evaluación del uso de las trampas GAT en 2020, en San Cristóbal y Santa Cruz.

\section{Métodos}

\section{Talleres de difusión}

Como parte del programa de vinculación con la comunidad auspiciado por la USFQ, se realizaron actividades para difundir información respecto a la transmisión del dengue y nuevas alternativas para monitorear las poblaciones del mosquito vector Ae. aegypti en las islas Galápagos, como se explica a continuación. Se 
presentó a la trampa GAT, como una opción de bajo costo y fácil de utilizar. En coordinación con el MSP y la Agencia de Regulación y Control de la Bioseguridad y Cuarentena para Galápagos (ABG), se organizó un taller informativo denominado “Taller teórico-práctico en Galápagos sobre el monitoreo y control del mosquito Aedes aegypti, vector del dengue, chikungunya y Zika, mediante el uso de trampas GAT (Gravid Aedes Trap)", en el que participaron actores claves y representantes de las diferentes organizaciones locales. Tanto al principio como al finalizar esta capacitación, se distribuyó un cuestionario con seis preguntas sobre el tema, para analizar y evaluar el impacto de esta actividad.

Adicionalmente, se efectuaron talleres de visitas domiciliares tanto en San Cristóbal como en Santa Cruz. El objetivo fue capacitar al personal local para colocar las trampas en las viviendas y realizar el monitoreo quincenal para recoger las muestras de mosquitos de las trampas. Se seleccionaron 10 viviendas en cada isla, con base en el criterio del personal del MSP, y estas sirvieron para colocar las trampas e iniciar el estudio piloto.

El taller de difusión de información se llevó a cabo en el Auditorio del Galápagos Science Center (GSC), de la USFQ, en San Cristóbal, el 7 de agosto de 2019. Asistieron el personal operativo y autoridades de varias instituciones, entre ellas MSP, ABG, el Municipio de Quito, la Policía Nacional, la Armada Nacional, el Municipio de San Cristóbal, el GAD de San Cristóbal y el Hospital Oskar Jandl (Figura 2).

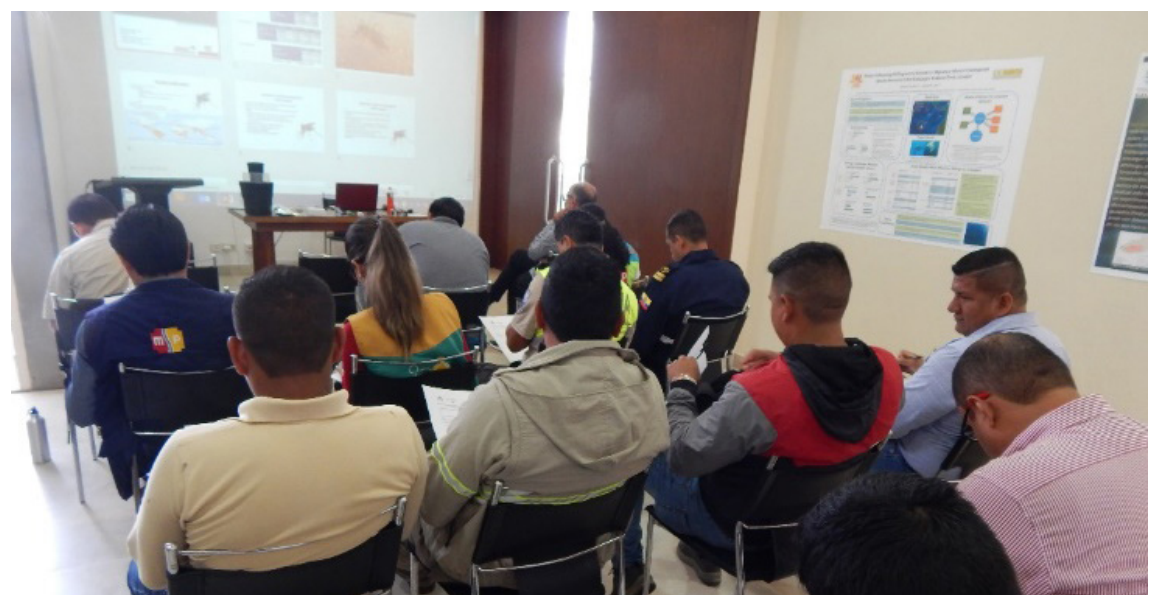

FIGURA 2. Capacitación sobre las trampas GAT realizada en el auditorio del GSC en Puerto Baquerizo Moreno, San Cristóbal el 7 de agosto de 2019 
Adicionalmente, se mantuvo una reunión el 8 de agosto de 2019 con la directora de ABG y su personal en la isla de Santa Cruz. Para estas actividades, se concretó la visita de los doctores Álvaro Eiras y Marcelo Carvalho de Resende, del Laboratorio Lintec de la UFMG, expertos en control vectorial (Figura 3a y 3b).
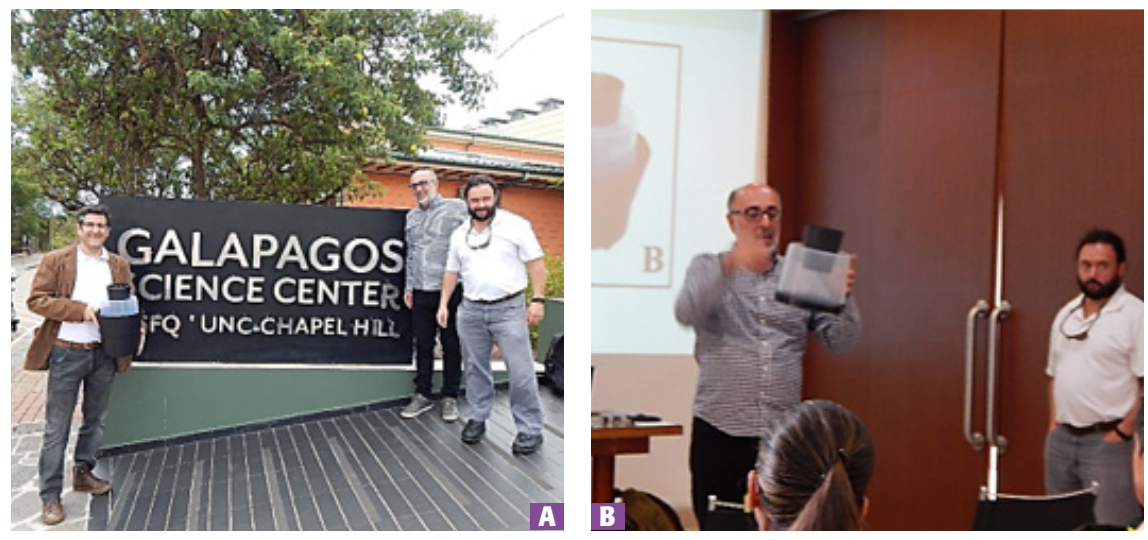

FIGURA 3. (a) Capacitadores del Taller de difusión. De izquierda a derecha: Dr. Álvaro Eiras (director del Instituto Lintec, UFMG, Brasil), Dr. Marcelo Calvalho de Resende (Lintec, UFMG) y Dr. Renato León, director del LEMMT, USFQ. (b). Charla del Dr. Carvalho de Resende acerca de las trampas GAT.

\section{Taller de visitas domiciliarias}

En San Cristóbal, se escogió al barrio Estación Terrena, en la ciudad de Puerto Baquerizo Moreno, para colocar 10 trampas GAT con la ayuda del personal del MSP. El taller se realizó el 6 de enero de 2020, cuando se seleccionaron 10 casas (con base en la presencia de lavanderías y tanques bajos, donde hay mayor riesgo de encontrar agua y criaderos de larvas de mosquitos), para explicar a la población sobre esta nueva iniciativa de monitoreo y colocar una trampa en cada patio trasero o zona del peridomicilio.

En Santa Cruz, y de acuerdo con la información preliminar del programa de control vectorial que lleva a cabo el MSP, se escogieron tres barrios de la ciudad de Puerto Ayora (Pampas Coloradas, Miraflores y Alborada) para colocar 10 trampas en casas seleccionadas con mayor riesgo de presencia de mosquitos. La actividad se llevó a cabo el 1 de febrero de 2020 (Figura 4a y 4b, Figura 5). 


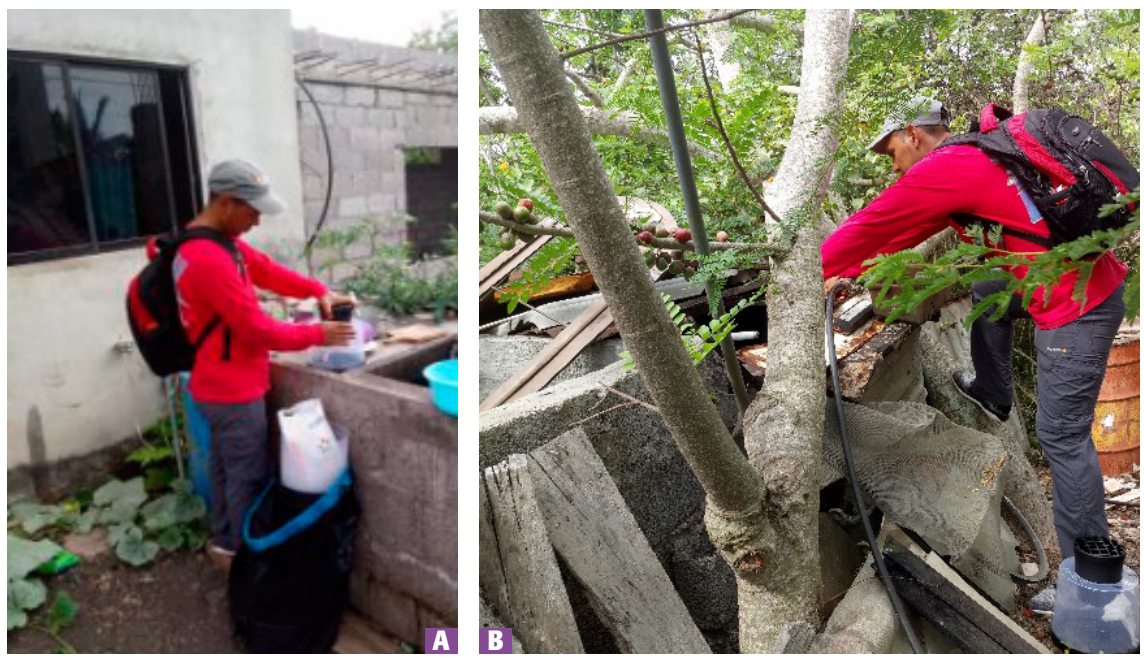

FIGURA 4. Personal de campo del proyecto ensamblan las trampas GAT (a) y las colocan en lugares estratégicos en el peridomicilio (b), durante los talleres de visitas domiciliarias en San Cristóbal y Santa Cruz.

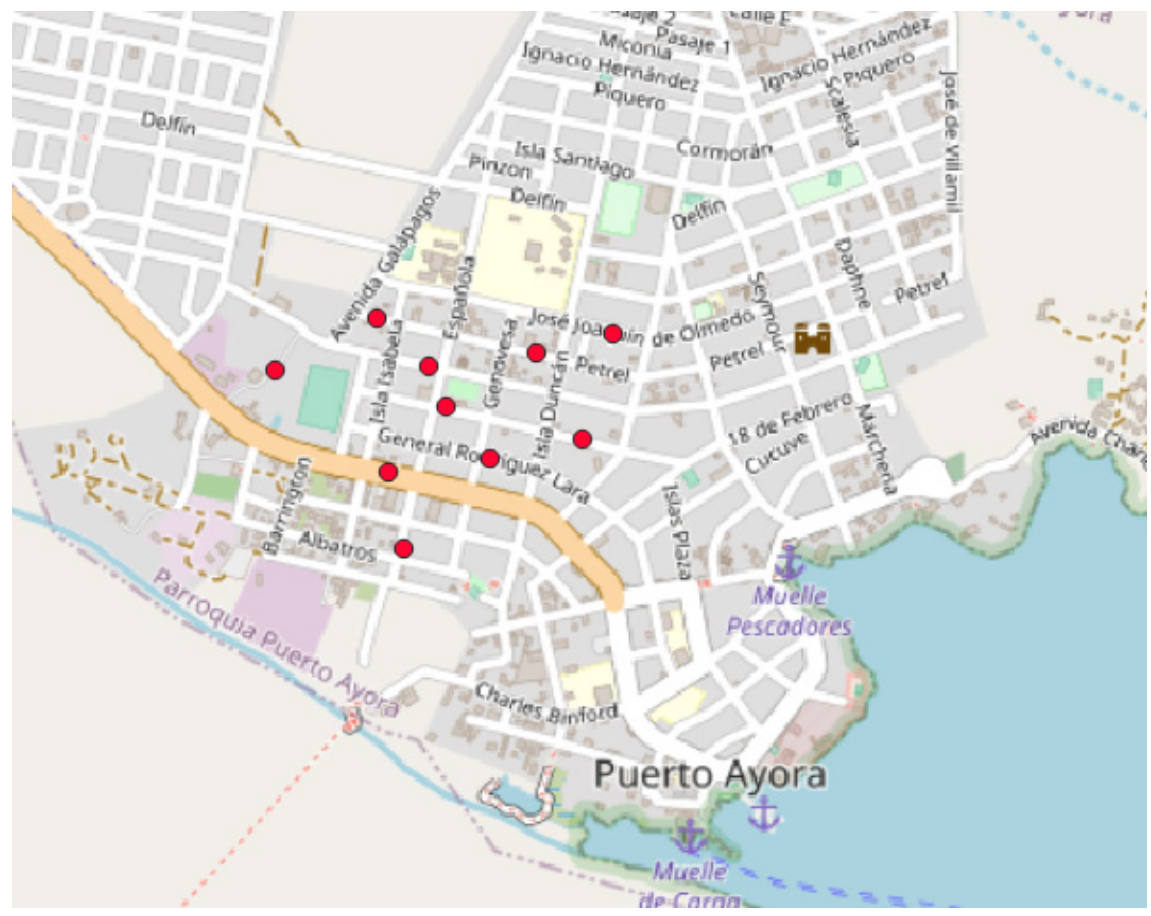

FIGURA 5. Lugares seleccionados (en rojo) donde se colocaron las trampas GAT en Puerto Ayora (isla Santa Cruz). 


\section{Ensamblaje de las trampas GAT y procesamiento de muestras}

Para que las trampas funcionen, se las debe lavar con agua para liberar cualquier olor a plástico que pueda repeler a los mosquitos. Antes de colocar la trampa, se añadió aproximadamente $500 \mathrm{ml}$ de agua potable al interior del recipiente plástico negro de la trampa, y luego se la armó. Para esto, sobre el recipiente plástico negro se colocó la segunda parte de la trampa, que es un cilindro cónico plástico transparente abierto a los dos lados. Del extremo con mayor diámetro del recipiente transparente, se acopla un anillo de plástico que permite sujetar una malla negra al extremo del diámetro mayor del recipiente transparente. A continuación, se arma la trampa colocando el recipiente transparente, con la malla acoplada, sobre el envase negro, de tal manera que la malla quede hacia abajo. Se coloca sobre la malla negra el atrayente químico, que viene en un pequeño recipiente plástico con tapa y papel aluminio (se mantuvo la tapa y se removió el papel aluminio de acuerdo con las instrucciones de Lintec, UFMG), y se cuelga de la pared del recipiente transparente por dentro, mediante un gancho, una tela blanca impregnada con el insecticida (Deltametrina), que matará a los mosquitos que ingresan en la trampa. Se termina de armar la parte superior de la trampa al colocar otro cilindro negro plástico de tamaño menor, que corresponde a la boca de la trampa. En la trampa ya armada, la malla en el interior evita el contacto de los mosquitos con el agua. Finalmente, se coloca una tapa de malla plástica en la boca de entrada en la parte superior de la trampa, que evita que otros insectos de mayor tamaño o basura entren accidentalmente a la trampa.

En las inspecciones quincenales, se recogió a los mosquitos de cada trampa GAT usando pinzas entomológicas, se los depositó en frascos plásticos adecuadamente rotulados y se los transportó al laboratorio del GSC de la USFQ. Se examinaron las muestras bajo un estereomicroscopio y se las identificó de acuerdo con claves taxonómicas. Los especímenes se almacenaron en etanol al 70\% o guanidina para analizarlos por biología molecular en el futuro.

\section{Estudio piloto con las trampas GAT}

Para obtener información preliminar de la eficiencia de las trampas GAT para colectar mosquitos Ae. Aegypti, se efectuó un estudio piloto en Santa Cruz y San Cristóbal, con 10 GAT en cada isla. Estas se colocaron en coordinación con el MSP en lugares claves en donde existiera mayor riesgo de presencia de mosquitos; se seleccionaron casas con lavanderías o agua estancada y que 
hubieran registrado presencia de mosquitos. El monitoreo pretendía usar la técnica estadística del cuadrado latino. De acuerdo con este modelo, cada cierto tiempo se relocalizan las trampas en una casa diferente; se rota en sentido de las manecillas del reloj entre casas, en calles en los cuatro lados de una manzana. El objetivo de rotar las trampas es minimizar la variación en las capturas ocasionadas por determinada trampa o por determinada vivienda. Sin embargo, esta metodología no fue aplicada por el tiempo limitado de evaluación

y las complicaciones logísticas debidas al inicio de la pandemia. Los resultados pretenden determinar la especificidad y la tasa de captura en cada sitio. E monitoreo se realizó quincenalmente por personal de campo que inspeccionó cada trampa y recogió las muestras de mosquitos para evaluar la densidad de población de estos vectores.

El monitoreo se llevó a cabo durante febrero y parte de marzo de 2020, sin embargo, fue suspendido por la pandemia. Los datos que se presentan son preliminares y corresponden a esas pocas semanas de colecta. El monitoreo entomológico continuará una vez que la emergencia sanitaria lo permita.

\section{Resultados y discusión}

\section{Talleres de difusión de información}

La capacitación fue evaluada y analizada. Todos los participantes coincidieron en que es importante llevar un control vectorial en las islas Galápagos (pregunta uno del cuestionario), basado sobre todo en el Ae. Aegypti, y que el cambio climático influye sobre la densidad de la población de vectores y la propagación de enfermedades vectoriales (pregunta cinco del cuestionario). Todos coinciden, además, en que puede ser importante usar las trampas GAT en Galápagos para controlar vectores como el Ae. aegypti. En el cuestionario que se aplicó después del taller, dos respuestas incluyen la importancia del monitoreo para detectar nuevas especies de mosquitos invasores, lo que es muy pertinente especialmente por las posibles futuras nuevas introducciones de esta especie desde Guayaquil y el riesgo de la introducción del $A e$. albopictus ya reportado en esta ciudad de la Costa [13]. 


\section{Resultados preliminares del estudio piloto}

Durante el periodo de estudio, se pudieron colectar tantos especímenes de Ae. aegypti, como de Ae. taeniorhynchus y de Culex quinquefasciatus en Puerto Baquerizo Moreno (San Cristóbal), mientras que solamente Ae. aegypti y Cu. quinquefasciatus fueron capturadas en Puerto Ayora (Santa Cruz). La tasa de positividad, es decir, el número de trampas positivas durante las colectas mostró variación tanto de semana a semana como de ciudad en ciudad, como se puede ver en las Figura 6.

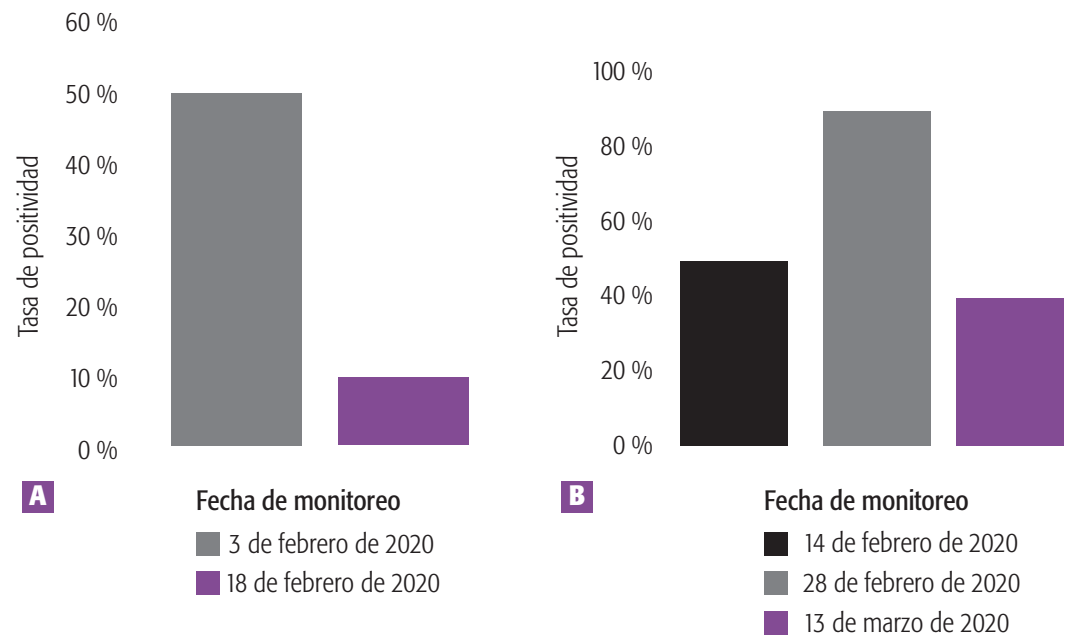

FIGURA 6. Tasa de positividad durante las búsquedas (a) en Puerto Baquerizo Moreno (San Cristóbal) y (b) en Puerto Ayora (Santa Cruz). Las barras representan el porcentaje de trampas que capturaron mosquitos durante cada fecha de recolección.

En San Cristóbal, las tasas de positividad encontradas fueron del 50\% para la primera colección y del 10\% para la segunda colección, esta tasa de positividad solo tiene en cuenta la captura o no de los especímenes. Durante el estudio, la tasa de positividad en Puerto Ayora fue del 50\%, 90\% y 40\%, para las tres colecciones, respectivamente. Esta tasa de positividad se mide por la captura o no de especímenes. Es importante decir que todas las trampas, excepto una, presentaron capturas en algún momento del estudio; una trampa en el barrio de Miraflores, en Santa Cruz, fue la única que no colectó mosquitos. 
Aunque este estudio piloto se llevó a cabo durante pocas semanas, los resultados preliminares sugieren que las trampas GAT pueden ser una buena alternativa para implementar el monitoreo vectorial de mosquitos adultos en las islas Galápagos. Las trampas pueden dejarse colocadas por largos periodos y no necesitan mayor mantenimiento; sin embargo, se requiere de personal local que inspeccione cada cierto periodo (entre una y dos semanas) y recolecte las muestras. Esto implica gasto de recursos y tiempo del personal de campo. Idealmente, personal del MSP o ABG puede realizar estas actividades si adopta esta metodología para complementar el control vectorial rutinario. Es necesario un proyecto con mayor número de trampas GAT para evaluar su uso sistemáticamente, el cual podría contribuir también al control vectorial. Se necesitan más estudios de evaluación, con miras a proponer la implementación de esta nueva metodología de monitoreo y control vectorial a las autoridades de salud locales en las islas Galápagos. 


\section{Bibliografía}

[1] Paupy, C., Le Goff, G., Brengues, C., et al. (2012). Genetic structure and phylogeography of Aedes aegypti, the dengue and yellow-fever mosquito vector in Bolivia. Infection, Genetics and Evolution, Journal of Molecular Epidemiology and Evolutionary Genetics in Infectious Diseases 12(6), 12601269. doi: https://doi.org/10.1016/j.meegid.2012.04.012.

[2] Rai, K S. (1991). Aedes albopictus in the Americas. Annual Review of Entomology (en prensa).

[3] Bonizzoni, M., Gasperi, G., Chen, X., \& James, A. A. (2013). The invasive mosquito species Aedes albopictus: current knowledge and future perspectives. Trends in Parasitology 29(9), 460-468.

[4] Jones, R., Kulkarni, M. A., Davidson, T., RADAM-LAC Research Team, \& Talbot, B. (2020). Arbovirus vectors of epidemiological concern in the Americas: A scoping review of entomological studies on Zika, dengue and chikungunya virus vectors. PloS one 15(2), e0220753. doi: https://doi.org/10.1371/journal. pone.0220753.

[5] Brown, J. E., Evans, B. R., Zheng, W., Obas, V., Barrera Martínez, L., Egizi, A., Zhao, H., Caccone, A., \& Powell, J. R. (2014). Human impacts have shaped historical and recent evolution in Aedes aegypti, the dengue and yellow fever mosquito. Evolution 68(2), 514-525.

[6] Brathwaite Dick, O., San Martín, J. L., Montoya, R. H., del Diego, J., Zambrano, B., \& Dayan, G. H. (2012). The history of dengue outbreaks in the Americas. The American journal of tropical medicine and hygiene 87(4), 584-593. doi: https://doi.org/10.4269/ajtmh.2012.11-0770

[7] García, E. (1953). Progresos en la lucha contra la fiebre amarilla en el Ecuador [Progress in the struggle against yellow fever in Ecuador]. Boletín de la oficina sanitaria panamericana. Recuperado de: https://iris.paho.org/ handle/10665.2/14686

[8] Ryan, S. J., Lippi, C. A., Nightingale, R., Hamerlinck, G., Borbor-Córdova, M. J., Cruz B, M., Ortega, F., León, R., Waggoner, E., \& Stewart-lbarra, A. M. (2019). Socio-ecological factors associated with dengue risk and Aedes aegypti presence in the Galápagos islands, Ecuador. International Journal of Environmental Research and Public Health, 16(5), 682.

[9] Real-Cotto, J. J., Regato Arrata, M. E., Burgos Yépez, V. E., \& Jurado Cobeña, E. T. (2017). Evolución del virus dengue en el Ecuador: Período 2000 a 2015 [Evolution of the dengue virus in Ecuador Period 2000 to 2015]. In Anales de la Facultad de Medicina 78(1), pp. 29-35. 
[10] MSP (Ministerio de Salud Pública). Dirección Nacional de Vigilancia Epidemiológica; Ministerio de Salud. (2020). Gaceta-Vectores SE 24/2020. Recuperado de: https://www.salud.gob.ec/gacetas-vectoriales-2020/

[11] Ponce, P., Morales, D., Argoti, A., \& Cevallos, V. E. (2018). First Report of Aedes (Stegomyia) albopictus (Skuse)(Diptera: Culicidae), the Asian Tiger Mosquito, in Ecuador. Journal of Medical Entomology 55(1), 248-249.

[12] Fundación Charles Darwin (Septiembre, 2020). Galapagos species Checklist. Recuperado de: https://www.darwinfoundation.org/en/datazone/ checklist?species=11720\#introduction

[13] Parra, F. (2014). Vigilancia de la salud: Registro histórico del dengue en la provincia de Galápagos [Health surveillance: Historic register of dengue in the province of Galapagos]. Quito: Ministerio de Salud Pública del Ecuador.

[14] León, R., Molina, C., Waters, W., Ortega, L. (2019). Aedes aegypti: Distribución y epidemiología del dengue, Zika y Chikungunya en Galápagos. Presentación oral al IV Simposio de Investigación y Conservación, SICG, GAIAS y GSC. 25 y 26 de junio de 2019.

[15] Asigau, S., Hartman, D. A., Higashiguchi, J. M., \& Parker, P. G. (2017). The distribution of mosquitoes across an altitudinal gradient in the Galapagos islands. Journal of Vector Ecology 42(2), 243-253.

[16] Tedjou, A.N., Kamgang, B., Yougang, A.P., Wilson-Bahun, T.A., Njiokou, F., \& Wondji, C.S. (2020). Patterns of Ecological Adaptation of Aedes aegypti and Aedes albopictus and Stegomyia Indices Highlight the Potential Risk of Arbovirus Transmission in Yaoundé, the Capital City of Cameroon. Pathogens 9, 491.

[17] Eiras, A. E., Buhagiar, T. S., \& Ritchie, S. A. (2014). Development of the gravid Aedes trap for the capture of adult female container-exploiting mosquitoes (Diptera: Culicidae). Journal of medical entomology 51 (1), 200-209. doi: https://doi.org/10.1603/me13104.

[18] Eiras, A., Resende, M., Acebal, J. \& Paixão, K. (2018). New Cost-Benefit of Brazilian Technology for Vector Surveillance Using Trapping System. 10.5772/ intechopen.78781.doi: http://dx.doi.org/10.5772/intechopen.78781

[19] Eiras, A. E., Pires, S. F., Staunton, K. M., Paixão, K. S., Resende, M. C., Silva, H. A., Rocha, I. G., Oliveira, B. A., Peres, A. M., Drumond, B. P., \& Ritchie, S. A. (2018). A high-risk Zika and dengue transmission hub: virus detections in mosquitoes at a Brazilian university campus. Parasites \& vectors 11(1), 359. doi: https:// doi.org/10.1186/s13071-018-2883-8. 